\title{
Shock Tube Testing and Modelling of Annealed Float Glass
}

\author{
Karoline Osnes ${ }^{1,2, *}$, Tore Børvik ${ }^{1,2}$, and Odd Sture Hopperstad ${ }^{1,2}$ \\ ${ }^{1}$ Structural Impact Laboratory (SIMLab), Department of Structural Engineering, NTNU, NO-7491 Trondheim, Norway \\ ${ }^{2}$ Centre for Advanced Structural Analysis (CASA), NTNU, NO-7491 Trondheim, Norway
}

\begin{abstract}
Failure of glass is dominantly brittle, and is caused by microscopic flaws randomly distributed on the surface. Fracture mainly initiates in these flaws, and this leads to a high variability in the glass strength, which depends on geometry, boundary conditions and loading situation. Consequently, the identification of the fracture strength, in e.g. finite element analyses, is not straightforward. For rapid loading conditions, as for blast loading situations, the glass strength is generally increased because flaws need time to grow into cracks. The current study aims to identify the probabilistic fracture strength of glass plates under blast loading as a function of the plate?s boundary conditions, geometry and loading by using a newly proposed strength prediction model. To validate this model in some measure, 12 blast tests on annealed float glass were performed in a shock tube. As expected, the tests showed a large scatter in fracture strength. The strength prediction model captured the main trends found in the experimental tests, but a closer investigation of the strain rate sensitivity of glass was deemed necessary. Finally, the results from the strength prediction model were used as input in a simulation of annealed float glass under blast pressure in the finite element program IMPETUS Afea Solver. By use of a node splitting technique, the simulations captured the behaviour displayed in the experimental tests to a great extent.
\end{abstract}

\section{Introduction}

Annealed float glass is largely used in window systems, and is a brittle material known to possess a large scatter in fracture strength. This is due to the existence of microstructural flaws, which are located on the surface of the glass plates [1]. Fracture normally initiates in these flaws when tensile stresses are applied, and the fracture strength can therefore be described as a combination of the flaw properties and the applied stress [2]. Consequently, the fracture strength is dependent on both the geometry, the boundary conditions and the loading situation of the plate. It can therefore be quite challenging to identify the fracture strength for use in e.g. finite element analyses [3]. In most Finite Element (FE) codes, failure modelling is based on a deterministic approach [4]. In other words, the chosen fracture strength (either given by a fracture stress or strain) applies to the entire glass plate. If this approach is to be used in a design process of glass, the fracture strength must be carefully chosen. It would naturally be advantageous to know the probability of the fracture strength specified in the FE model. The current study aims to determine this probability as a function of the glass plate's geometry, boundary condition and loading situation. The proposed numerical method is based on the recent work by Yankelevsky [5], but includes some additional features and adjustments. The probabilistic model, as in the work by Yankelevsky, is based on the existence of microscopic surface flaws in glass, and uses Monte Carlo simulations to predetermine the fracture strength for glass plates in a

\footnotetext{
*e-mail: karoline.osnes@ ntnu.no
}

given scenario. The model will here be referred to as the strength prediction model [6].

To investigate if the strength prediction model is able to provide realistic results, 12 blast tests on annealed float glass [6] were performed in the SIMLab Shock Tube Facility [7]. The results from these tests are compared to the results provided by the model. Finally, the calculated probabilistic fracture stress is used as input in a simulation of a blast loaded glass plate. The FE program IMPETUS Afea Solver is used, as it enables an element-separation, or node-splitting method. This method is highly advantageous when it comes to modelling of problems including brittle fracture and fragmentation [8].

\section{Experimental programme}

\subsection{Material}

The glass plates used in the current experimental programme are made out of clear annealed soda-lime-silica glass. The mechanical behaviour of glass is elastic up to the point of failure, which normally occurs due to crack propagation of existing microscopic surface flaws. This happens when stresses (higher than a given threshold) are applied normal to the flaws, so that they open and grow. The strength of the glass is naturally dependent on the properties of the flaws, but also on the environment [9] and the rate of loading. Generally, the glass strength increases with the loading rate because flaws need time to grow into cracks [3]. The stiffness of the glass, however, is relatively insensitive to the loading rate [10]. Table 1 
lists some commonly used material parameters for sodalime-silica glass. The parameters $\rho, E, v$ and $K_{I C}$ refer to the density, Young's modulus, Poisson ratio [11], and fracture toughness for mode I loading (i.e., opening of a crack), respectively. The value of $K_{I C}$ is reported in [12], and is based on the work by Widerhorn [9].

Table 1. Material parameters for soda-lime-silica glass.

\begin{tabular}{cccc}
$\left(\mathrm{kg} / \mathrm{m}^{3}\right)$ & $(\mathrm{MPa})$ & & $(\mathrm{MPa} \sqrt{m})$ \\
\hline 2500 & 70000 & 0.2 & 0.75
\end{tabular}

\subsection{Blast loading}

An idealised loading history for a blast pressure can be viewed in Figure 1. Typically, it consists of a positive and a negative pressure phase, and has an instant rise to peak reflected pressure $P_{\max }$ from atmospheric pressure $P_{0}$ at the arrival time $t_{\mathrm{a}}$. The duration of the positive and the negative phase is $t_{\mathrm{d}+}$ and $t_{\mathrm{d}-}$, respectively. The minimum value of the negative pressure is $P_{\mathrm{s}}$. In the blast tests pre-

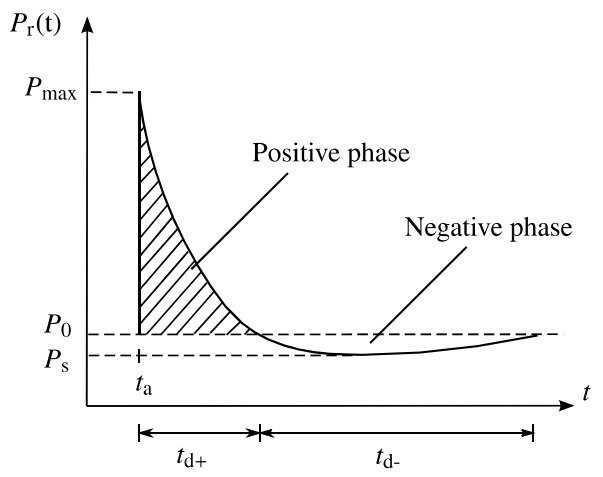

Fig. 1. Idealised pressure time history for the reflected blast wave from an explosion [13].

sented in this study, the negative pressure phase is negligible, and the pressure time history can be represented by the modified Friedlander equation [13] stated as

$$
P_{r}(t)=P_{0}+P_{\text {max }}\left(1-\frac{t-t_{a}}{t_{d+}}\right) \exp \left(\frac{-b\left(t-t_{a}\right)}{t_{d+}}\right)
$$

where, $b$ governs the curvature from $P_{\max }$.

\subsection{SIMLab Shock Tube Facility}

As an alternative to explosive detonations, the SIMLab Shock Tube Facility (SSTF) was used to subject plated glass specimens to blast pressure loading. The SSTF has earlier proven to produce planar and uniform pressure loading, and is a reliable and safe option for blast testing. A detailed description of the SSTF and how the blast pressure is produced can be found in the work by Aune et al. [7]. Figure 2 shows an illustration of the SSTF. It is divided into a tank, and a high-pressure and a low-pressure chamber, denoted the driver and the driven, respectively. The test specimen is mounted inside the tank at the end of

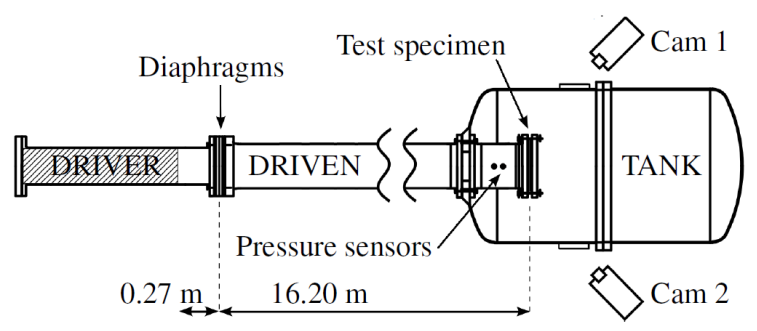

Fig. 2. Sketch of the SIMLab Shock Tube Facility (SSTF) seen from above. Adapted from [7].

the driven. To measure the applied blast pressure on the specimen, data from two pressure sensors placed $245 \mathrm{~mm}$ and $345 \mathrm{~mm}$ away from the plate are fitted to Equation (1) and interpolated back to the arrival time $t_{\mathrm{a}}$. See [7] for details. The deformation of the specimen is obtained in 25 different points, which are placed with a distance of $60 \mathrm{~mm}$ $\mathrm{c} / \mathrm{c}$ over the glass surface. These points, denoted optical targets, are tracked by a point-tracking procedure available in the in-house three dimensional digital image correlation (3D-DIC) code eCorr [14]. Images from two synchronised Phantom v1610 high-speed cameras are used for this purpose. The recording rate of the cameras is set to $24 \mathrm{kHz}$. The setup for blast testing of glass plates in the SSTF is

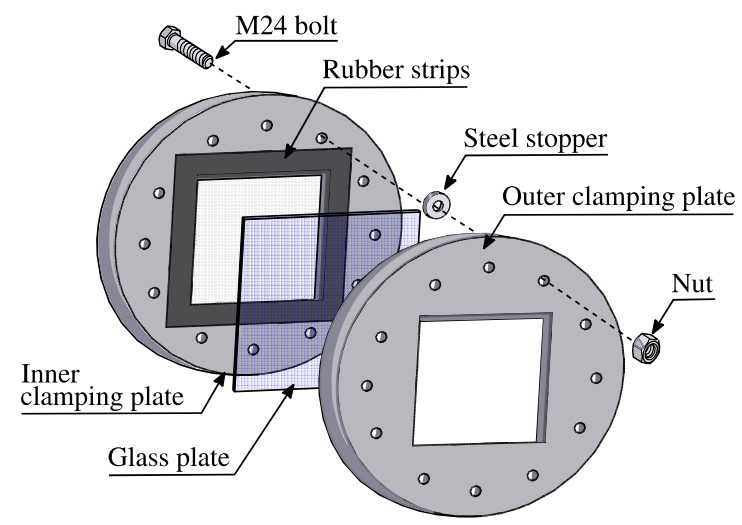

Fig. 3. The custom-made fastening system used in the blast experiments on glass in the SSTF. The setup is here disassembled and shows one out of 12 bolts, stoppers and nuts.

partly shown in Figure 3. The glass is clamped between two $25 \mathrm{~mm}$ thick aluminium plates, denoted the inner and outer clamping plate. The inner clamping plate is fastened to the end of the driven, and the blast wave will therefore travel in the direction from the inner to the outer clamping plate. Neoprene rubber strips (with a thickness of $4 \mathrm{~mm}$,

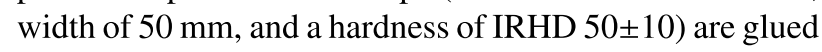
to the clamping plates and placed on each side of the glass. In order to properly fasten the outer clamping plate, while limiting the prestressing of the glass, steel stoppers are placed on the bolts that connect the two clamping plates together. In this way, the bolts can be firmly tightened, and the contraction of the rubber will be minimal. 


\subsection{Results}

In total, twelve blast tests on $400 \mathrm{~mm} \times 400 \mathrm{~mm} \times 3.8 \mathrm{~mm}$ float glass were performed in the SSTF. A summary of the results from the tests is shown in Table 2 [6]. The parameters $P_{\max }, t_{\text {frac }}, D_{\text {max }}, v_{\text {frag }}$ and $i_{+}$refer to the maximum reflected overpressure, the time of fracture initiation, the maximum centre displacement before fracture, the maximum measured fragment velocity, and the impulse of the positive phase before fracture, respectively. The column denoted Fracture specifies whether the fracture initiated at the face (f) or the boundary (b) of the glass. The tests are divided into three classes (A, B and C), based on the level of maximum reflected overpressure on the glass. As can

Table 2. Summary of the blast tests on float glass. Note that $t=$ 0 corresponds to the time of arrival $t_{a}$ of the blast wave.

\begin{tabular}{c|cccccc} 
Test & $P_{\max }$ & $t_{\text {frac }}$ & $D_{\max }$ & $v_{\text {frag }}$ & $i_{+}$ & Fracture \\
\hline Unit & $\mathrm{kPa}$ & $\mathrm{ms}$ & $\mathrm{mm}$ & $\mathrm{m} / \mathrm{s}$ & $\mathrm{kPa} \cdot \mathrm{ms}$ & - \\
\hline $\mathrm{A}-01$ & 53.0 & 1.25 & 4.61 & 15.8 & 60.2 & $\mathrm{~b}$ \\
$\mathrm{~A}-02$ & 51.6 & 1.38 & 5.18 & 14.7 & 64.0 & $\mathrm{~b}$ \\
\hline $\mathrm{B}-01$ & 63.7 & 1.00 & 3.31 & 24.5 & 58.7 & $\mathrm{f}$ \\
$\mathrm{B}-02$ & 64.6 & 1.21 & 5.51 & 18.8 & 71.4 & $\mathrm{~b}$ \\
$\mathrm{~B}-03$ & 65.5 & 1.46 & 5.75 & 17.3 & 86.2 & $\mathrm{~b}$ \\
$\mathrm{~B}-04$ & 62.5 & $\times$ & 5.56 & $\times$ & 293.9 & $\times$ \\
$\mathrm{B}-05$ & 63.3 & 1.00 & 1.50 & 21.8 & 58.4 & $\mathrm{f}$ \\
$\mathrm{B}-06$ & 62.9 & 0.96 & 3.71 & 19.3 & 56.2 & $\mathrm{~b}$ \\
$\mathrm{~B}-07$ & 64.0 & 1.13 & 5.79 & 19.0 & 66.6 & $\mathrm{~b}$ \\
$\mathrm{~B}-08$ & 62.9 & $\times$ & 5.98 & $\times$ & 294.2 & $\times$ \\
\hline $\mathrm{C}-01$ & 73.4 & 1.29 & 6.01 & 21.5 & 86.5 & $\mathrm{~b}$ \\
$\mathrm{C}-02$ & 73.2 & 1.33 & 2.26 & 22.3 & 88.9 & $\mathrm{~b}$
\end{tabular}

be seen from Table 2, only two out of the 12 glass plates withstood the subjected blast pressure. Since this did not apply to the "class A" tests, the test series clearly illustrates the stochastic nature of fracture in glass. In most of the tests, the fracture initiated at the boundary, i.e., under the rubber strips. An illustration of how the fracture typically develops when initiating at the face versus the boundary is shown in Figure 4. Note that for the case in Figure 4b, the fracture most likely initiated at the back of the plate, i.e., the surface faced away from the cameras. The reason is that at the boundary, tensile stresses occur at the back surface.
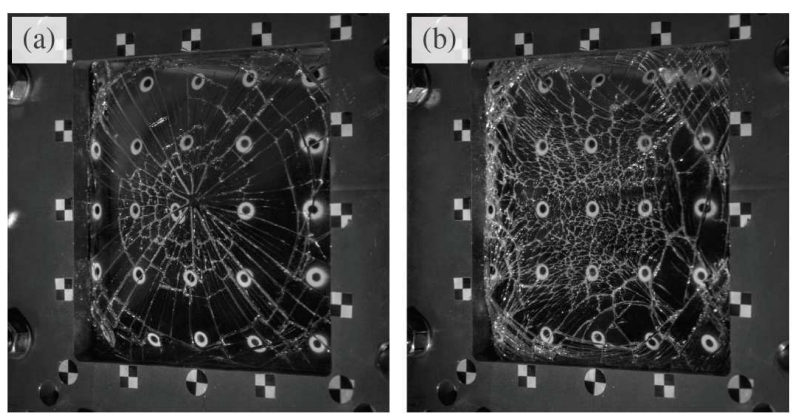

Fig. 4. Photo of a fractured glass plate at $t=4.2 \mathrm{~ms}$ for tests: (a) B-01 (face fracture initiation), (b) B-02 (boundary fracture initiation).

\section{Numerical modelling}

\subsection{Strength prediction model}

The following presents a stochastic model for predicting fracture initiation in glass plates. The model, referred to as the strength prediction model, is based on the work by Yankelevsky [5], but includes some additional features and adjustments [6].

The strength prediction model is based on the fact that fracture in glass plates generally initialise in pre-existing microscopic surface flaws. When the glass surface is subjected to tensile stresses, the flaws will open and grow. In the strength prediction model, failure is defined as the onset of unstable crack growth in one flaw. The following failure criterion is thus adopted

$$
K_{I} \geq K_{I C}
$$

Here, $K_{I}$ is the stress intensity factor for mode I loading, i.e., the opening of a crack, and $K_{I C}$ is the critical value, or the fracture toughness. The surface flaws are assumed to be elliptical shaped, and the following empirical expression [15] can be employed

$$
K_{I}=\frac{\lambda_{\mathrm{s}} f(\phi)}{\sqrt{Q}} \sigma \sqrt{\pi a}
$$

Here, $a$ is the depth of the crack, $\sigma$ is the remote tensile stress normal to the crack, and $Q$ is the flaw shape parameter calculated as

$$
Q=1+1.464\left(\frac{a}{c}\right)^{1.65}
$$

where $c$ is the half-length of the crack. Further, $\lambda_{\mathrm{s}}$ is the surface correction factor defined by

$$
\lambda_{\mathrm{s}}=\left[1.13-0.09\left(\frac{a}{c}\right)\right]\left[1+0.1(1-\sin \phi)^{2}\right]
$$

and $f(\phi)$ is an angular function depending on $\phi$

$$
f(\phi)=\left[\sin ^{2}(\phi)+\left(\frac{\mathrm{a}}{\mathrm{c}}\right)^{2} \cos ^{2}(\phi)\right]^{\frac{1}{4}}
$$

The parameter $\phi$ defines the angle of a point on the elliptic crack, where $\phi=0$ is the critical value. For rapid loading conditions, the following failure criterion is adopted instead of Equation (2)

$$
\frac{1}{\tau} \int_{t-\tau}^{t} K_{I}(\bar{t}) d \bar{t} \geq K_{I C}
$$

Here, $\tau$ is denoted the incubation time, or the microstructural fracture time. This is explained as the minimum time required to initiate crack growth, and is included to avoid that spurious peaks of stress result in premature failure of the glass.

For a given loading scenario, plate geometry and boundary conditions, the stress state of a glass plate (without fracture initialising) can be calculated by using the finite element method, or if possible, analytical expressions. The strength prediction model employs the known 
stress state throughout a given loading scenario, including information about the surface flaws to calculate information such as the probability distribution of fracture stresses and/or forces, position of fracture initialisation, and a failure percentage. This is done through Monte Carlo simulations of a sufficient number of glass plates. Each of the glass plates is given a so-called flaw map, i.e., the distribution of surface flaws including their size, shape, location and orientation. In each analysis the failure criterion given by either (2) or (7) is checked for all the assigned flaws throughout the entire loading scenario.

The input required from a user of the strength prediction model is the following:

- The stress state in a glass plate (on the surface)

- Fracture toughness $K_{I C}$

- Flaw shape $a / c$

- Maximum flaw depth $a_{\max }$

- Flaw density $\rho_{\text {flaw }}$

- Number of plates to analyse $n_{\text {plate }}$

- Size of a jumbo plate $A_{\text {jumbo }}$

The jumbo plate is a large plate from which the analysed plates are cut. The majority of glass used for windows is cut from plates with nominal lengths of 4500 , 5100 or $6000 \mathrm{~mm}$, and widths equal to $3210 \mathrm{~mm} \mathrm{[16].} \mathrm{It} \mathrm{is}$ further assumed that a jumbo plate includes only one flaw with a depth of $a_{\max }$. An exponential flaw depth distribution is adopted [5], which is discretised as

$$
a_{i}=a_{\max }\left(1-\frac{\ln \left(N_{i}\right)}{\ln \left(N_{0}\right)}\right), \quad N_{i}=R_{1}\left(N_{0}-1\right)+1
$$

here, $N_{0}$ is the number of flaws, $N_{i}$ is the number of flaws with depths larger than $a_{i}$, and $R_{1}$ refers to a random variable uniformly distributed on $[0,1]$. The flaw orientations are further given by

$$
\alpha_{i}=R_{2} \pi
$$

as it is believed that the flaws do not favour any orientation. $R_{2}$ is a random variable uniformly distributed on $[0,1]$.

\subsection{Strength prediction of blast loaded plates}

The strength prediction model is further used in an attempt to find the strength distribution of the blast tests presented in Section 2.4. The parameters presented in Table 3 are employed. The value of $a / c$ is based on the work by Levengood [17], while both $\rho_{\text {flaw }}$ and $a_{\text {max }}$ are adopted from the work by Wereszczak et al. [18]. As for the value of $n_{\text {plate }}$, Yankelevsky [5] stated that a number of 5000 plates would result in a converged probability distribution.

To obtain the stress state of a glass plate (before fracture) in the blast tests, the FE software Abaqus [19] was employed. However, other FE codes could also have been used. The FE model itself consisted only of the glass plate (modelled with shell elements) and the rubber strips
Table 3. Input parameters for the strength prediction model.

\begin{tabular}{l|l}
$K_{I C}$ & $0.75 \mathrm{MPa} \sqrt{\mathrm{m}}$ \\
$a / c$ & 1 \\
$a_{\text {max }}$ & $100 \mu \mathrm{m}$ \\
$\rho_{\text {flaw }}$ & 2 flaws $/ \mathrm{cm}^{2}$ \\
$n_{\text {plate }}$ & 5,000 \\
$A_{\text {jumbo }}$ & $3210 \mathrm{~mm} \times 6000 \mathrm{~mm}$ \\
$\tau$ & $10 \mu \mathrm{s}$
\end{tabular}

(modelled with solid elements). The aluminium clamping plates, stoppers and bolts were indirectly included in the model by restricting the movement of the outer rubber surfaces. The glass material was modelled as linear-elastic with the first three parameters in Table 1. The rubber was also modelled as linear-elastic, with a Young's modulus of $2 \mathrm{MPa}$ and Poisson's ratio of 0.46 . To ensure that the test setup was properly modelled, the deformations in the glass plate from a selected test without failure (B-04) were compared with a corresponding Abaqus simulation. Three optical targets (nine points when disregarding symmetry) were tracked, which are denoted P0, P1 and P2. The resulting time-displacement history, together with an illustration of the optical targets, are presented in Figure 5. It shows a good correlation of the test and simulation, and the model could therefore be used to acquire the stress state. The following presents some results obtained from

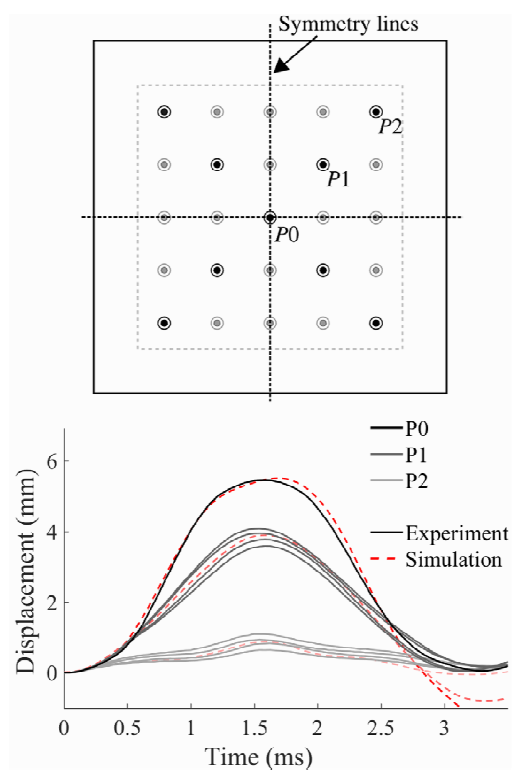

Fig. 5. Displacement from experiment and Abaqus simulation in points P0-P2 for test B-04. Illustration of P0-P2 included.

the strength prediction model. Figure 6 shows the location of the fracture initiation (distinguished between front and back failure) for test A-01. Compared to the actual shock tube tests, the location of front failures agreed well, as fracture initiation in the tests always occurred within the area proposed by the strength prediction model. Whether the position of back failure is in agreement with the tests or not was difficult to determine as the fracture initiation occurred under the rubber strips. 
Table 4 presents the failure percentage and time of fracture initiation of tests A-01, B-04 and C-01. If we compare the results with the experiments, both the total and the front failure percentage are overestimated by the strength prediction model. However, to draw any definite conclusions, we need a much larger number of experimental tests. Nevertheless, there are possible reasons for this discrepancy, such as the glass being damaged by the sharp aluminium edges of the inner clamping plate. It may also be due to dynamic effects not being sufficiently accounted for in the model. Regarding the time of fracture initiation, the experimental results for class $\mathrm{A}, \mathrm{B}$ and $\mathrm{C}$ all lie within or are equal to the predicted extreme values. The proba-

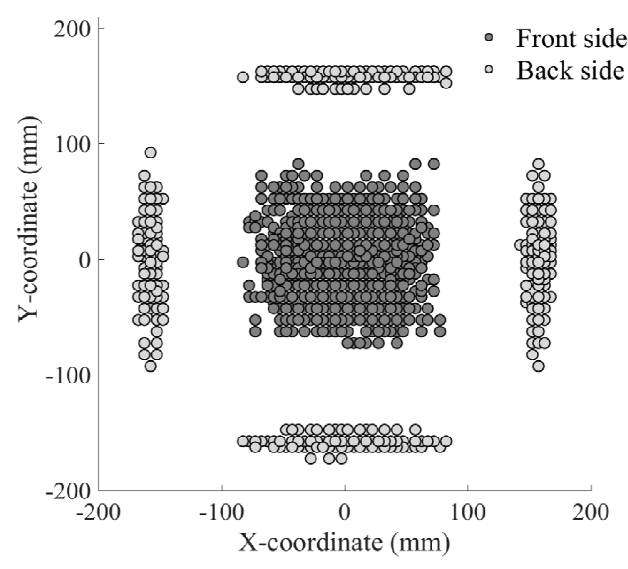

Fig. 6. Location of fracture initiation for test A-01 visualised as a surface map. The origin is at the plate's centre.

Table 4. Failure percentages and time of fracture initiation $t_{\text {frac }}$.

\begin{tabular}{c|cc|cc} 
& \multicolumn{2}{|c|}{ Failure- $\%$} & \multicolumn{2}{c}{ Time of fracture initiation $(\mathrm{ms})$} \\
\hline Test & Total & Back side & Front side & Back side \\
\hline A-01 & 94.2 & 19.8 & $0.88-1.92$ & $0.95-1.7$ \\
B-04 & 99.8 & 4.62 & $0.82-1.71$ & $0.85-1.54$ \\
C-01 & 100 & 3.71 & $0.76-1.41$ & $0.75-1.33$
\end{tabular}
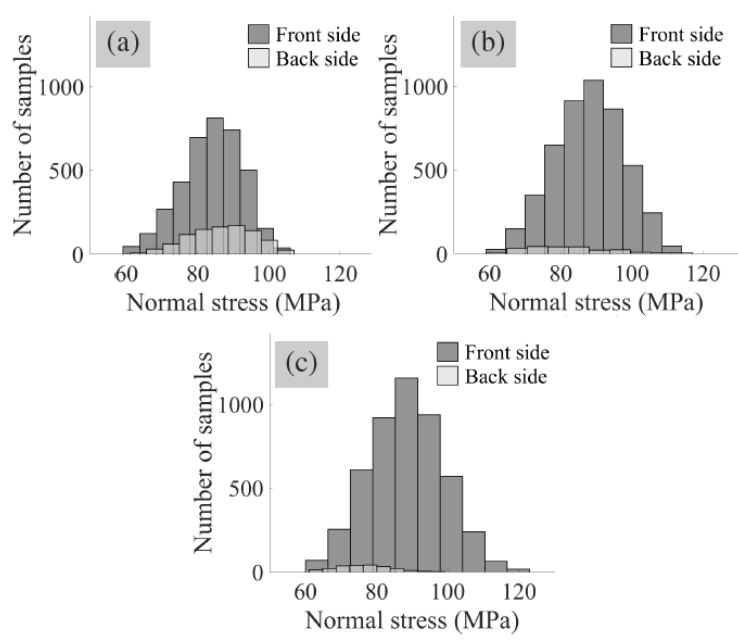

Fig. 7. The distribution of normal stresses at failure determined by the strength prediction model for tests (a) A-01, (b) B-04, (c) C-01. bility distribution of normal stresses at failure, divided into front and back failures, are further shown in Figure 7 for tests A-01, B-04 and C-01. The ordinate refers here to the number of fictitious glass plates tested. The value of the normal stresses extends over values from about $60 \mathrm{MPa}$ to $120 \mathrm{MPa}$.

\subsection{FE modelling in IMPETUS Afea Solver}

The FE program IMPETUS Afea Solver was employed to simulate the fracture process of the glass plate in test B04 , i.e., a test where fracture initiated almost exactly at the centre. The code enables features including higher order elements and a node-splitting technique [20]. Node splitting facilitates a more realistic fracture pattern and crack propagation by separating the mesh at the element borders instead of deleting elements, as with the traditional element erosion technique. The FE model was built in a similar manner as for the Abaqus model, however, the rubber was modelled with $7 \mathrm{~mm} \times 7 \mathrm{~mm} \times 4 \mathrm{~mm}$ 64-node hexahedral elements, and the glass with $4 \mathrm{~mm} \times 4 \mathrm{~mm} \times$ $3.8 \mathrm{~mm} 40$-node pentahedron elements. Pentahedron elements were chosen to enable fracture in two additional directions. A brittle fracture criterion with the parameters $K_{I C}$ equal to $0.75 \mathrm{MPa} \sqrt{\mathrm{m}}$, and a fracture stress of $60 \mathrm{MPa}$ was included for the glass. The fracture stress was chosen as this was the most critical value found from the strength prediction model. It corresponds to a case where fracture initiates in a flaw of maximum size $a_{\max }$.

Figure 8 compares pictures from the experiment and the simulation, and shows that the behaviour is highly similar. For a more quantitative comparison, the displacement in points P0-P2 is shown in Figure 9. Note that the displacement in point $\mathrm{P} 2$ was no longer trackable after $4 \mathrm{~ms}$, and is therefore not displayed after this point. Again, it is seen that the agreement between the simulation and the experiment is very good, and illustrates that the use of node splitting enables a highly realistic behaviour of fracture in glass. Naturally, as the fracture initiated almost in the centre of the glass plate in the experiment, the simulation captured the response more easily. This is because the fracture criterion yielded fracture initiation in the point of maximum principal stress, i.e., in the centre. For simulations involving boundary-fractured plates, one would need to trigger the fracture initiation at the boundary somehow to get a comparable response.

\section{Concluding remarks}

A model for predicting the fracture strength of glass plates, based on the existence of microscopic surface flaws, has been presented. In an attempt to validate the model, 12 blast tests on annealed float glass were conducted in the SIMLab Shock Tube Facility. As expected, the tests illustrated the stochastic nature of fracture in glass. Further, the strength prediction model successfully captured trends shown in the tests. However, a slight overestimation of the total failure percentage and the failure percentage of 

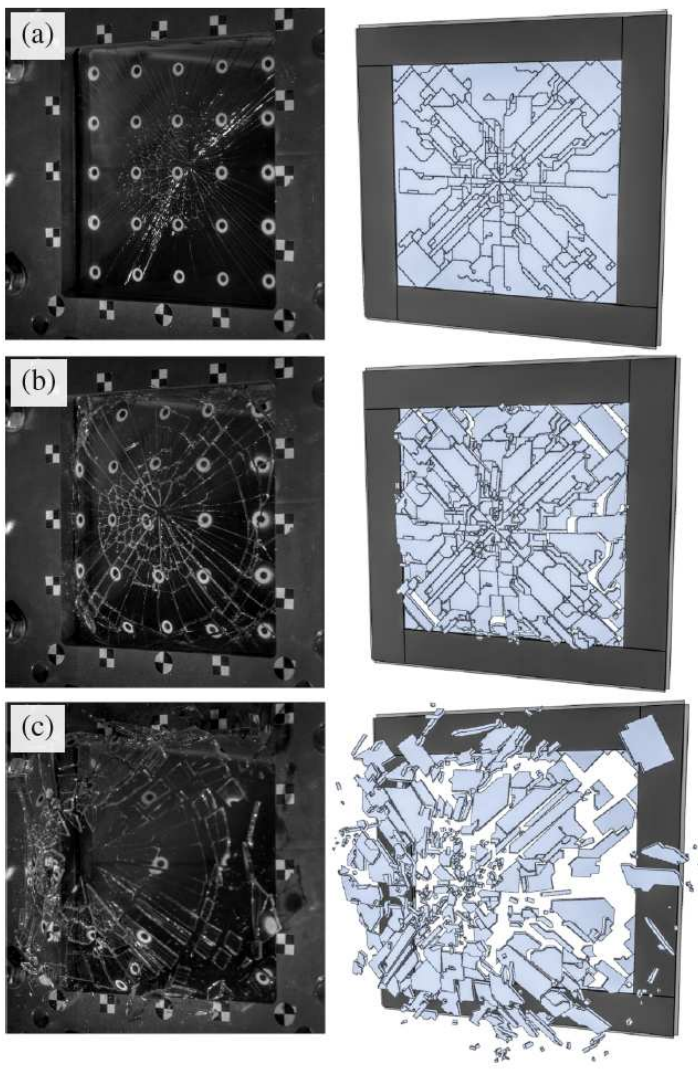

Fig. 8. Pictures of the fractured plate in the experiment and simulation at: (a) $1 \mathrm{~ms}$, (b) $4.2 \mathrm{~ms}$, (c) $10 \mathrm{~ms}$.

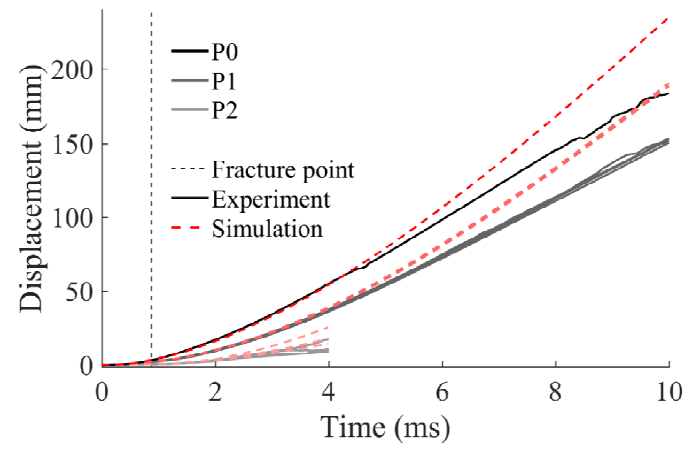

Fig. 9. Displacements in points P0-P2 for test B-04.

front-failure was shown for the specified loading scenarios. This may be due to dynamic effects not being sufficiently accounted for in the model, and a strain rate dependent fracture toughness might need to be included. It should, however, be noted that the number of experimental tests was rather limited for validation purposes. Finally, results from the strength prediction model were employed as input for a brittle fracture model in the finite element program IMPETUS Afea Solver. By using a node splitting method, the behaviour in the simulation was highly comparable with experiments.

The work has been carried out with financial support from CASA (project number 237885), Centre for Research-based Innovation (CRI), at the
Norwegian University of Science and Technology (NTNU). The authors wish to acknowledge Mr. Trond Auestad and Mr. Tore Wisth for assistance with the experimental programme. We would also like to thank Modum Glassindustri AS for providing glass specimens for use in the experiments.

\section{References}

1. W.L. Beason, J.R. Morgan, Journal of Structural Engineering 110, 197 (1984)

2. J.B. Wachtman, W.R. Cannon, M.J. Matthewson, $M e-$ chanical properties of ceramics, 2nd edn. (John Wiley \& Sons, 2009)

3. M. Larcher, G. Solomos, F. Casadei, N. Gebbeken, International Journal of Impact Engineering 39, 42 (2012)

4. J. Pelfrene, J. Kuntsche, S. Van Dam, W. Van Paepegem, J. Schneider, International Journal of Impact Engineering 88, 61 (2016)

5. D.Z. Yankelevsky, Engineering Structures 79, 244 (2014)

6. K. Osnes, T. Børvik, O. Hopperstad, (Submitted for possible journal publication) (2018)

7. V. Aune, E. Fagerholt, M. Langseth, T. Børvik, International Journal of Protective Structures 7, 340 (2016)

8. J.K. Holmen, J. Johnsen, O.S. Hopperstad, T. Børvik, European Journal of Mechanics-A/Solids 55, 221 (2016)

9. S.M. Wiederhorn, Journal of the American Ceramic Society 52, 99 (1969)

10. X. Zhang, Y. Zou, H. Hao, X. Li, G. Ma, K. Liu, International Journal of Protective Structures 3, 407 (2012)

11. NS-EN 572-1: Glass in building - Basic soda-lime silicate glass products - Part 1: Definitions and general physical and mechanical properties, CEN (2012)

12. G. Anstis, P. Chantikul, B.R. Lawn, D. Marshall, Journal of the American Ceramic Society 64, 533 (1981)

13. D. Cormie, G. Mays, P. Smith, Blast effects on buildings, 2nd edn. (Thomas Telford Publishing, 2009)

14. E. Fagerholt, T. Børvik, O. Hopperstad, Optics and Lasers in Engineering 51, 299 (2013)

15. J. Newman, I. Raju, Engineering Fracture Mechanics 15, 185 (1981)

16. NS-EN 572-1: Glass in building - basic soda-lime silicate glass products - part 2: Float glass, CEN (2012)

17. W. Levengood, Journal of Applied Physics 29, 820 (1958)

18. A.A. Wereszczak, M.K. Ferber, W. Musselwhite, International Journal of Applied Glass Science 5, 16 (2014)

19. Abaqus, Version 6.14 (Dassault Systèmes Simulia Corporation, Providence, RI, USA, 2014)

20. IMPETUS Afea Solver, http://www.impetus-afea. com/, accessed: 2018-01-20 